\title{
Cytochemical study of the distribution of RNA and DNA in the synaptonemal complex of guinea-pig and rat spermatocytes
}

\author{
R. Ortiz', O.M. Echeverría ${ }^{1}$, E. Ubaldo ${ }^{1}$, A. Carlos ${ }^{1}$, C. Scassellati² ${ }^{2}$ G.H. Vázquez-Nin ${ }^{1}$ \\ 'Laboratory of Electron Microscopy, Department of Cell Biology, Faculty of Sciences, UNAM, Apartado Postal 70- \\ 438, México D.F. México and ${ }^{2}$ Center of Electron Microscopy, University of Lausanne, 27 rue Bugnon, CH-1005 \\ Lausanne, Switzerland
}

Accepted: 6/3/2002

Key words: mammalian testis, ribonucleoprotein, meiosis, in situ hybridization, electron microscopy

\section{SUMMARY}

The distribution of DNA and RNA in the synaptonemal complex and related structures, was studied using high resolution cytochemical methods and in situ hybridization, in guinea pig and rat testis. Serial sectioning demonstrates that frequently the formation of the synaptonemal complex (SC) occurs without a previous development of isolated chromosomal axes. The lateral elements of the forming SC are in continuity with pairs of DNA-containing thin filaments. These chromatin filaments fold in numerous short loops just before incorporating to the lateral elements. Some of these loops are included in the ribbon-like structure of the lateral elements of the mature SC. We propose that these short loops contain the DNA attachment sequences associated with the proteins of the LE. During the formation of the SC one of the two chromatin filaments incorporates at the central surface of the forming lateral element (LE) and the other is located at the external side of the LE. This unexpected distribution does not correspond to the pair of thick filaments previously discerned in structure of the LE. The presence of RNA associated with the DNA-containing thin filaments, as well as with the axial chromatin elements of the forming SC, may be related with the transcription occurring during meiotic prophase, specially during zygotene stage. We propose that RNA is involved in a still uncharacterized process essential for pairing.

\section{INTRODUCTION}

The synaptonemal complex (SC) was observed for the first time by Watson (1952) in rat spermatocytes as filamentous structures, and described more accurately four years later by Moses (1956) in a crayfish and by Fawcett (1956) in the pigeon. In few years, structures corresponding to SCs were found in the meiotic prophase of protists, fungi, plants and animals (see review by Westergard and Wettstein, 1972). Three-dimensional reconstructions of complete nuclei of pachytene spermatocytes demonstrated that the total number of SC per nucleus is equal to the haploid number of chromosomes of the species, allowing the conclusion that this structure corresponds to a bivalent (Wettstein and Sotelo, 1967). The classical hypothesis that SC is related with crossing-over formation was supported by: a) its constant presence in meiotic prophase and their absence when pairing does not take place; b) its extension all along each bivalent; c) the regular

Correspondence to: G.H. Vásquez-Nin

E-mail: ghvn@hp.fciecias.unam.mx 
occurrence of the bridging system (see review by Sotelo, 1969). The presence of short stretches of SC in relation with chiasma in diplotene stage, also suggested a specific role for the SC in homologous exchanges (Westergaard and Wettstein, 1972). However, some findings cast some doubts on its relationships with chiasma development: the pairing space contains little or no DNA, and the SC occurs in circumstances other than orthodox pairing (Sotelo, 1969). The finding of recombination nodules provided support for the notion that SC is involved in chiasma formation (Carpenter, 1975, 1979 a, b). Posterior studies on the meiosis of yeast using synchronized cultures (Kleckner et al., 1991), and fluorescent in situ hybridization (Scherthan et al., 1992), demonstrated that in this group, double strand breaks (DSB) and recombination occur before synapsis and SC formation (Kleckner, 1996; Padmore et al., 1991; see review by Hawley and Arbel, 1993). Experiments with meiotic mutants showed that the alteration of DSB prevents the formation of a normal SC (Bishop et al., 1992; Roeder, 1995; Baker et al., 1995). These results support the view that in yeast the SC is not involved in recombination and that its formation depends on previous DSB and recombination. However, electron microscopical studies demonstrated that in Drosophila females meiotic mutants eliminating crossing over and gene conversion do not alter the formation of the SC (McKim et al., 1998).

Immunocytochemical localization of the DNA at the level of electron microscope in mouse and rat spermatocytes and chick oocytes, demonstrated the presence of significant amounts of DNA in the lateral elements (LEs). The labeling of the central space is very low except for few specific sites: the recombination nodules and few fibrils not related to these nodules (Vázquez-Nin et al., 1993).

However, the precise location of the two sister chromatids in the SC is not yet known. As stated several years ago, the key to the knowledge of cytophysiological role of the SC is to find out the distribution of DNA in the SC (Sotelo, 1969).

RNA has not been related to recombination or other possible functions of the SC. Nevertheless, the presence of RNA in the LEs of the SC was suggested by Wolstenholme and Meyer (1966) using optical histochemistry and electron microscopy. The use of a preferential staining procedure for ribonucleoprotein (RNP)-containing structures in electron microscopy, indicated the presence of RNP in the
LEs of the synaptonemal complex, as well as in leptotene and zygotene axial cores (Esponda and Stocker, 1971; Vázquez-Nin and Echeverría, 1976). Furthermore, hydrolyses with RNase were found to cause profound alterations of the LEs (Vázquez-Nin and Echeverría, 1976). However, the precise distribution of RNA-containing structures associated with the forming SC, has not been studied.

The aim of the present work is to study the distribution of both nucleic acids during the formation and the maturity of the SC.

\section{MATERIALS AND METHODS}

Testes of young adult guinea pigs and rats were used in all experiments.

The standard procedure for preparing the samples involves fixation with $2.5 \%$ glutaraldehyde in 0.2 M phosphate buffer $\mathrm{pH} 7.3$ for $1 \mathrm{~h}$ at room temperature, rinsing in the same buffer, dehydration with ethanol, and embedding in an epoxy resin (glycide ether 100, Merck, Darmstadt, Germany).

One micrometer thick sections were stained with toluidine blue. The cellular associations present in the seminiferous tubules were studied at light microscope and compared with the classical descriptions of the spermatogenic cycle of the guinea pig (Clermont, 1960) and of the rat (Leblond and Clermont, 1952).

In order to stain RNP structures, the uranyl acetate-EDTA-lead citrate (Bernhard, 1969) procedure was used. DNA was stained using the osmium-ammine procedure (Cogliati and Gautier, 1973) modified by Vázquez-Nin and coworkers (1995).

\section{Immunolocalization}

Samples of seminiferous tubules were fixed in 2$4 \%$ paraformaldehyde in $0.15 \mathrm{M}$ phosphate buffer pH 7.3 and embedded in Lowicryl K4M. Polymerization was carried out using UV at $-20{ }^{\circ} \mathrm{C}$. The ultrathin sections were mounted in Formvar coated nickel grids, and processed for postembedding immunolocalization as described previously (Biggiogera et al., 1989; Vázquez-Nin et al., 1990).

\section{Ultrastructural in situ hybridization}

Specimens were fixed in $2 \%$ paraformaldehyde with $0.2 \%$ glutaraldehyde added and embedded in Lowicryl K4M. 
Genomic DNA from guinea pig liver and spleen was labeled with digoxigenin-11-dUTP by nick translation according with the manufacturer's protocol (Boehringer Mannheim, Germany).

The in situ hybridization was performed on the grids with the denatured probe with or without preannealing for $24-48 \mathrm{~h}$ at $37-45^{\circ} \mathrm{C}$. The probe was used at $10 \mathrm{ng} / \mathrm{ml}$ final concentration in a hybridization solution containing $10 \%$ deionized formamide, $10 \%$ Dextran sulfate and 1X saline sodium citrate buffer $(0.15 \mathrm{M} \mathrm{NaCl}, 0.015 \mathrm{M}$ sodium citrate; $\mathrm{pH}$ 7.0). The DNA in the specimen was not denatured. The hybrids were detected by incubation of the grids on a drop of mouse anti-digoxigenin (Boehringer, Mannheim, Germany). The mouse anti-digoxigenin was detected by a rabbit anti-mouse IgG (Aurion, Wageningen, The Netherlands).

In order to confirm the specificity of the hybridization signal, some sections were pre-treated with $2 \mathrm{mg} / \mathrm{ml}$ ribonuclease (type IA, Sigma) in $1 \mathrm{mM}$ triethanolamine-acetic acid buffer, $\mathrm{pH} 7,3$ for $8 \mathrm{hr}$. at $37^{\circ} \mathrm{C}$.

All preparations were contrasted with $2.5 \%$ uranyl acetate for $5 \mathrm{~min}$ and lead citrate for $1 \mathrm{~min}$.

\section{RESULTS}

\section{General Morphology}

To describe the organization of the SC it is necessary to situate the sections in relation to the axes of the complex. The frontal plane contains lateral and central elements lengthwise. The transverse plane is perpendicular to the main axis of the SC. Lateral plane is parallel to this axis and perpendicular to the frontal plane. It contains some, but not all the elements of the SC. This nomenclature has been used by Moses (1968) and Sotelo (1969).

Serial sectioning of the nuclei of spermatocytes in early meiotic prophase demonstrated the presence of short SC between 0.5 and $1.8 \mathrm{~mm}$ in length. One extremity of some of them is in contact with nuclear envelope, but others are distant from the nuclear periphery (Fig. 1). The reconstruction of 29 SC of 10 different nuclei showed that the ends of the lateral elements of most of these short SC are not in continuity with converging axial elements. Continuities of the lateral elements of the forming SC with a pair of thin filaments can be seen when both structures are contained in the thickness of one section (Figs. 2, 3). Frontal sections show that one of these filaments is incorporated in the external side of the lateral element and the other at the central side, that is in the border of the pairing space (Fig. 2). The pairs of thin filaments and the LE are surrounded by loops of different length and filaments of chromatin that correspond to long loops not contained in the section (Fig. 3). The frequency of short loops (40 to $200 \mathrm{~nm}$ in length, measured from the tip to the axial filament) increases in the proximity of the forming chromosomal axis or lateral element of the growing SC. These short loops are
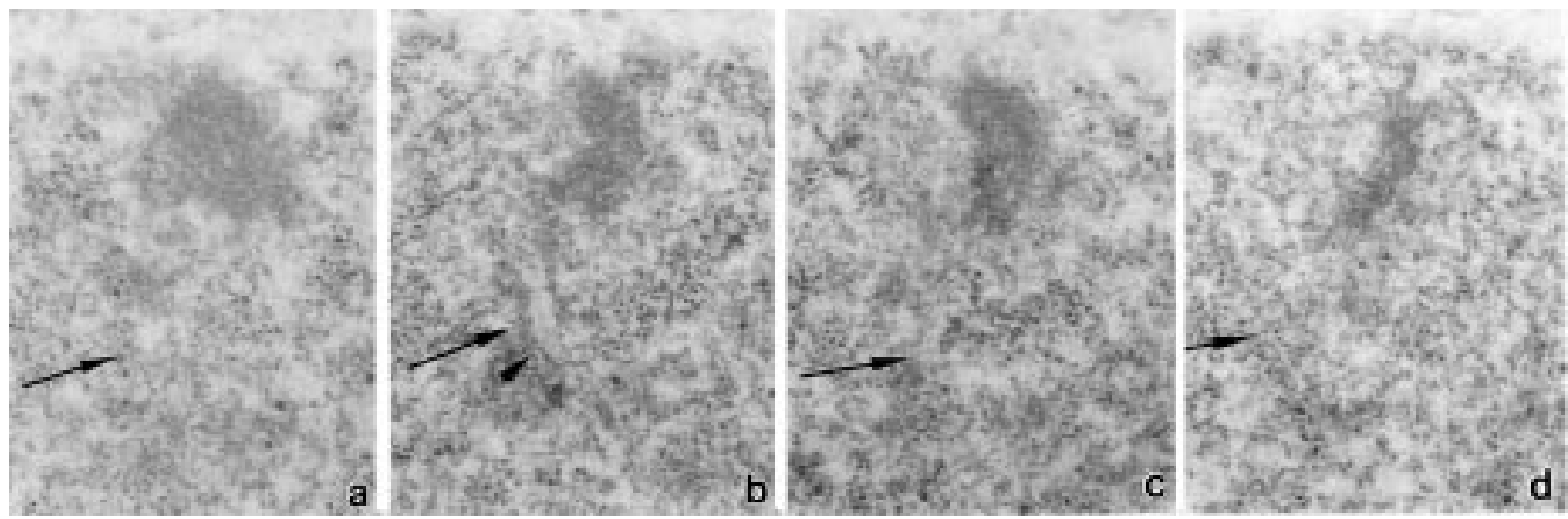

Fig. 1 - Serial sections of a synaptonemal complex in the process of formation. The arrows indicate the position of the synaptonemal complex. Figure a. No synaptonemal complex can be seen. Figure b. The three elements of the synaptonemal complex are depicted. The lower tip of the lateral elements are apparently in continuity with aligned structures (arrow head). Figures c and $d$. No structure related to the forming synaptonemal complex is visible. The complex is $530 \mathrm{~nm}$ in length, no contact with the nuclear envelope or continuity with converging axial elements can be seen. X 28,500. 


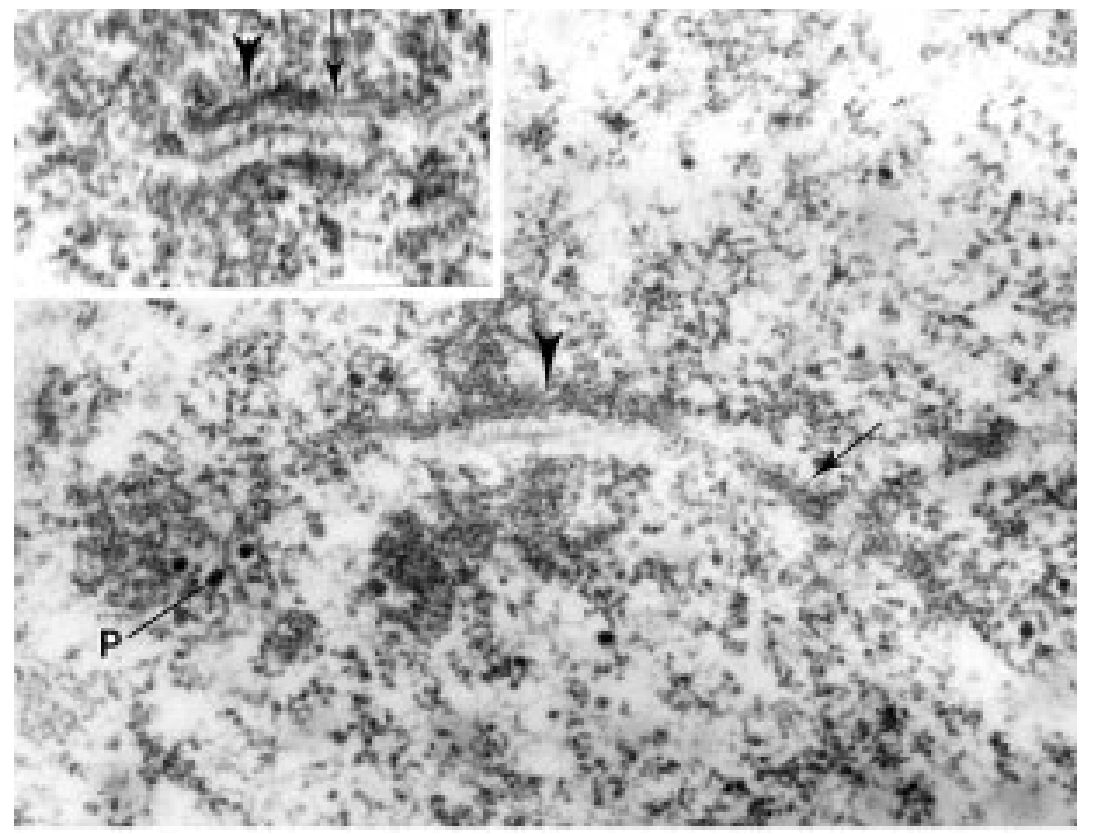

Fig. 2 - Forming synaptonemal complex. General staining. A continuity between the fibers of the lateral element (arrow heads) and a pair of filaments (arrow), can be seen. $\mathrm{p}$ - perichromatin granules. X 40,000. Inset. The external and central filaments of the forming lateral element (arrow head) are clearly continuous with the precursor fine filaments (arrow). X 55,000.

closely distributed in quasi-periodic form along the filament (Fig. 3 inset). The period is about 22



Fig. 3 - Zygotene. The formation of the chromosome axis is simultaneous with final pairing. The lateral element (A) is continuous with two fine filaments (M). The central space of the forming synaptonemal complex is visible as a chromatin exclusion zone (arrow). F - filaments of chromatin surrounding the complex. X40,000. Inset. Numerous short loops form a quasi-periodic structure (arrow head). X26,500.
$\mathrm{nm}(\mathrm{X}=22.3, \mathrm{SD}=3.98, \mathrm{~N}=87)$. It was estimated from measures carried out in 24 LEs of 8 nuclei.

The LEs of mature SCs present a transverse striation (Fig. 4) with a period about $20 \mathrm{~nm}(\mathrm{X}=20.5$, $\mathrm{SD}=2.39$, 36 LEs were measured in 10 nuclei). The lateral views show that the LEs of pachytene $\mathrm{SC}$ are longitudinal striated ribbons about $0.2 \mu \mathrm{m}$ wide, in which some thick irregular bundles of fibrils can be discerned, but no cross striation can be seen (Fig, 5).

Frontal sections demonstrate the presence of a dark stained filament of irregular thickness in the border between central space and the LEs. This filament frequently appears as beads on a string or aligned beads (Fig. 5 inset).



Fig. 4 - Pachytene. General staining. A lateral element of a synaptonemal complex showing a periodic transverse striation. X74,000. 


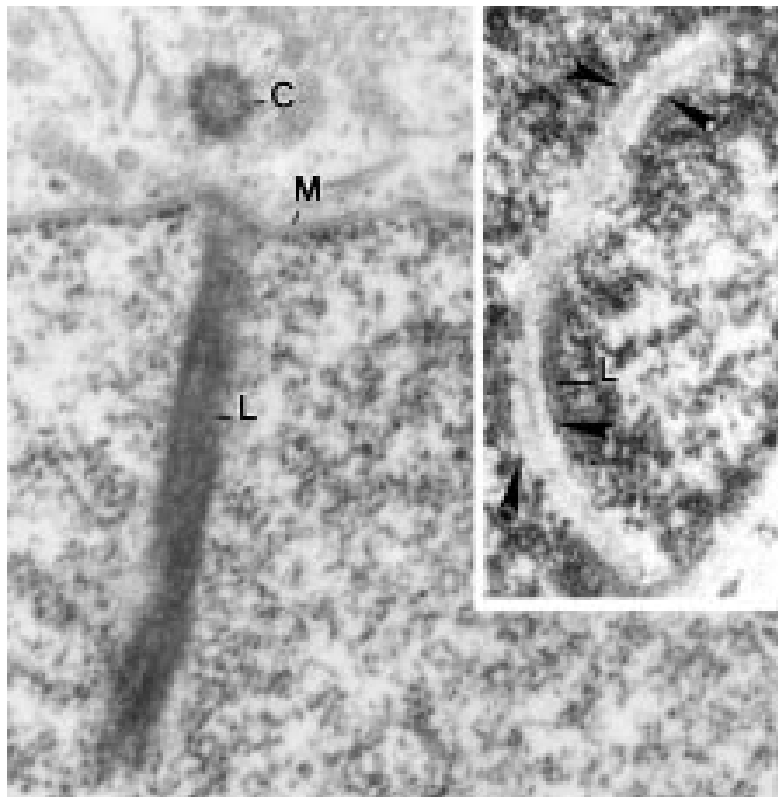

Fig. 5 - Pachytene. General staining. Lateral view of the ribbon-like lateral element (L) of a synaptonemal complex. Longitudinal ill defined bundles of fibrils can be seen. No periodic pattern can be discerned. C- centriole. M- nuclear envelope X34,500. Inset. Frontal section of a synaptonemal complex. Portions of a dark stained filament (arrow heads) can be seen in the central side of the lateral elements (L). X37,000.

\section{Distribution of RNP}

The lateral elements of the mature pachytene SC are faintly stained with EDTA procedure. A row of small granules positively contrasted with this staining method can be seen in contact with the medial face of the LEs of the SC in frontal sections (Fig. 6). The forming chromosome cores and the developing LE are frequently darkly stained. The fine folding fibrils found in continuity with the growing end of these structures are also positively contrasted with EDTA procedure. These fibrils present short loops as those depicted with standard contrast techniques (Fig. 6 inset).

The fibrils and granules surrounding the SC are intensively labeled by in situ hybridization of a genomic probe carried out without denaturation of the DNA of the sample (Fig. 7). The internal face of the LE is also labeled. The sections treated with RNase before hybridization present few or no gold grains (data not shown).

\section{Distribution of DNA}

Osmium-ammine procedure specific for DNA contrasts positively the fine filaments aligned in pairs.

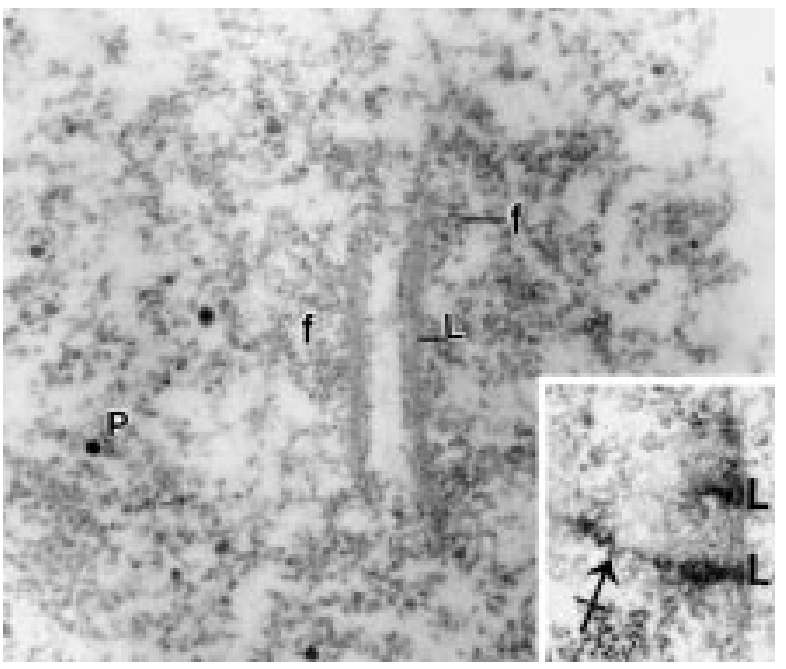

Fig. 6 - Advanced pachytene. EDTA staining. The lateral elements are stained clear gray (L). A row of dark stained granules can be seen in the central surface of the lateral elements. The central space is devoid of RNP structures. Numerous perichromatin fibrils (f) are located in the vicinity of the synaptonemal complex and to a lesser extent in the rest of nucleoplasm. Perichromatin granules (p) are dark stained. $\mathrm{X} 37,000$. Inset. Two forming axial elements aligned in the vicinity of the nuclear membrane are dark stained (L). The crossed arrow indicates the continuity of a forming axial element with a dark stained fibril depicting short folds. X24,000.

These filaments correspond to those found in continuity with the lateral elements of the SC in preparations stained with general contrasting method. The loops of different length surrounding the fine filaments are also contrasted with osmium-ammine. Short loops are specially abundant (Fig. 8).

Important changes in the distribution of chromatin take place at the moment of the formation of the SC. The central space is almost empty of DNA-containing fibrils, except for few transverse fibrils of chromatin. A longitudinal fibril located to the central surface of the LE is positively contrasted with osmium ammine staining method (Figs. 9, 10). This fibril corresponds to the filament of irregular diameter showed by general staining (Fig 5 inset), and to the row of small granules found with EDTA procedure for RNP (Fig. 6). Oblique sections of the LE demonstrate the presence of numerous short DNA-containing loops and fibrils inside the LE (Figs. 10, 11). Some of the fibrils crossing the LE are continuous with the chromatin surrounding the SC (Figs. 9, 10). 




Fig. 7 - In situ hybridization using a genomic probe to detect RNA. General staining. Pachytene stage. Numerous RNP fibrils and granules located in the nucleoplasm are labeled. Gold grains are also associated with fibrils surrounding a synaptonemal complex (arrows). The boundary between the lateral element and the central space is also labeled (arrow heads). X74,000.

\section{DISCUSSION}

The distribution of DNA in the mature pachytene SC has been studied by Coleman and Moses (1964) using of indium trichloride staining complemented with DNase hydrolysis. They conclud-

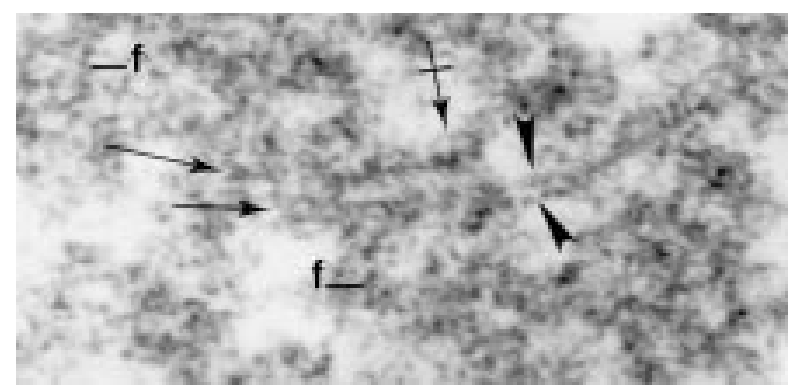

Fig. 8 - Osmium-ammine staining specific for DNA. Zygotene stage. Two aligned fine filaments are positively contrasted (arrows). The two filaments composing the forming lateral elements are clearly depicted (arrow heads). At this stage the fine filaments are folded forming short loops (crossed arrow). Abundant fibers of extended chromatin are present in the nucleoplasm (f). X75,000.

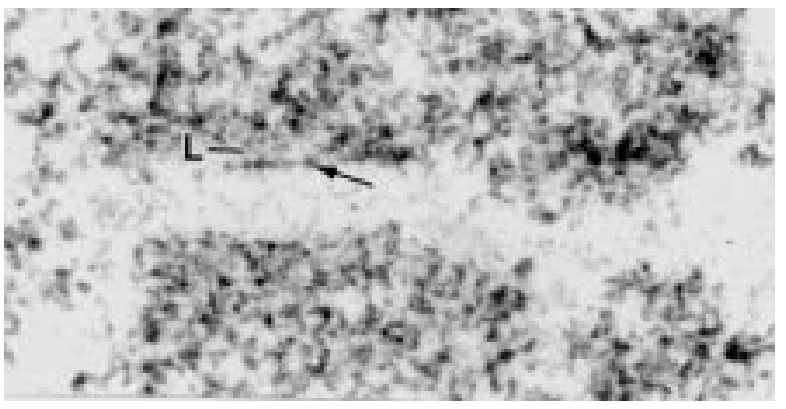

Fig. 9 - Pachytene. Osmium-ammine staining specific for DNA. An irregular filament located in the central side of the lateral element is dark stained (arrow). The pairing space is devoid of chromatin. No precise external limit of the lateral element (L) can be seen. X80,000.

ed that there is few or no DNA in the central element and that the central space is traversed by some fibrils containing DNA. These results were supported by a study using immunolocalization of DNA at the level of the electron microscope (Vázquez-Nin et al., 1993).

The use of the osmium-ammine method allowed us a precise and specific determination of the distribution of the DNA-containing structures in forming and mature SC. The present results demonstrate that, in rat and guinea-pig spermatocytes, each lateral element of the SC in the process

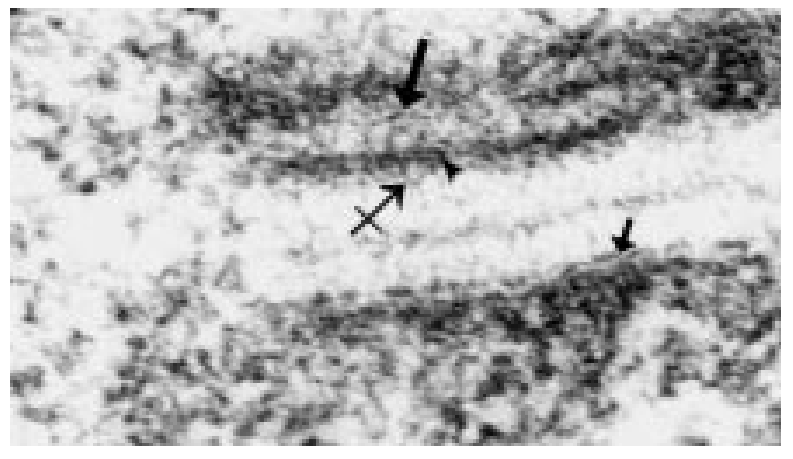

Fig. 10 - Pachytene. Osmium-ammine staining. Both lateral elements of the synaptonemal complex are sectioned at different inclination probably due to a turn of the complex. The beads on a string aspect of the filament located in the central side of the lateral element is clearly depicted (short arrow). The thicker regions of these filaments are dark stained. The filaments of the surrounding chromatin are continuous with those of the LE. The upper LE is cut along the wider surface of the ribbon. Short loops protrude to the pairing space (crossed arrow). Numerous fibrils and short loops lay in the thickness of the LE (long arrow). The external border of the element indicated by a denser arrangement of the filaments of chromatin. X70,000. 


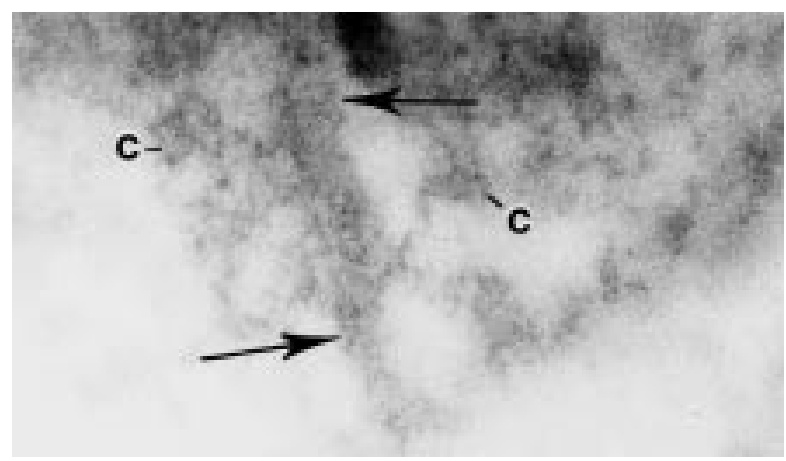

Fig. 11 - Pachytene. Osmium-ammine staining. Lateral view of a LE. Positively contrasted short loops can be seen immersed in a ribbon-like region stained clear gray (arrows). $\mathrm{C}$ - filaments of the chromatin surrounding the complex. X57,000.

of formation is frequently continuous with a pair of thin DNA-containing filaments folded in loops of different length representing the sister chromatids of one of the homologous chromosomes.
One of these filaments is located at the medial side of the LE, while the other is folded in numerous loops at the external face of the LE. The presence of a chromatid axis at the internal side and the other at the external side of the LE is an unexpected distribution, its cytophysiological interpretation is open to discussion in terms of the opportunities of each filament to become involved in different meiotic processes. If the SC is essential for the initiation of recombination, in organisms other than yeast as was classically proposed (see review by Hawley and Arbel, 1993), the proximity of each chromatid with the recombination nodules located in the central space may influence its opportunity to become involved in a recombination event. Nevertheless, the filament located at the external side of the LE may gain access to the medial space by means of the inward oriented loops or by changing positions with the filament situated at the internal side of the LE. However, if in mam-

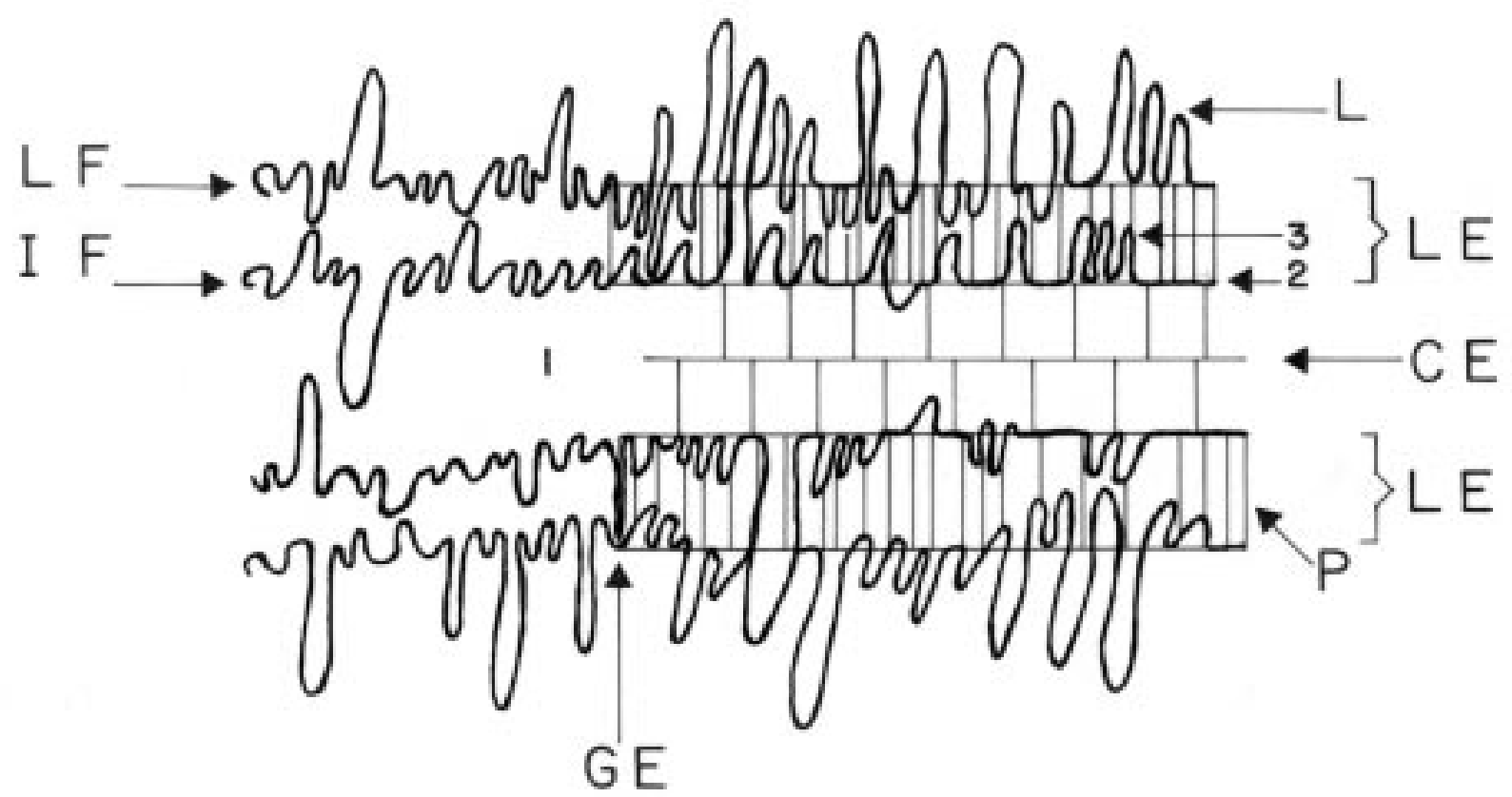

Fig. 12 - Schematic drawing of a frontal view of a forming synaptonemal complex showing the distribution of some of its macromolecular components. The lateral elements (LE) extend to the right of the growing end (GE) of the synaptonemal complex. At a short distance from the GE the central element (CE) and the transversal filaments appear. The lateral thin DNA-containing filament (LF), situated to the left of the growing end, is continuous with the filament located in the external side of the lateral element. The loops of these filaments are associated with RNA-containing structures. The internal DNA-containing filament (IF) is continuous with the filament located in the internal border of the lateral element (2). These filaments are also associated with RNP. Numerous DNA-containing loops (3) are located inside the protein structure $(\mathrm{P})$ of the lateral elements. Proteins form cross striation in a periodic pattern $(\mathrm{P})$ that can be seen in frontal section. The appearance of a zone of exclusion of DNA and RNA (1), takes place before the formation of the classical elements of the synaptonemal complex. 
mals the recombination is initiated before the SC formation as in yeast (Padmore et al., 1991; Sym et al., 1993; Borde et al., 2000), the association of the chromatids with the both sides of the LE may be only relevant to crossover interference and disjunction process (Maguire, 1995).

The two DNA-containing thin filaments are present in frontal sections of the SC. Doubleness of the LE has been observed in rat spermatocytes (Sotelo and Trujillo-Cenoz, 1958), in pigeon (Nebel and Coulon, 1962) and in various other animals and plants (see review by Moses, 1968). These pairs of thick fibers frequently found in lateral sections of the LE, were interpreted as representing the two chromatids of one chromosome (Nebel and Coulon, 1962; Baker and Franchi, 1967; Moses, 1968). The results of the present work indicate that in rat and guinea-pig the DNAcontaining filaments do not correspond to thicker fibers that can be discerned in the protein structure of the lateral elements.

The development of the SC is accompanied by an intense rearrangement of chromatin characterized by: 1) the formation of a region of almost complete exclusion of DNA and RNA, the central space, and 2) the folding of the fine filaments to form multiple short loops before incorporating into the SC. These short loops are included in the ribbon-like structure of the LE in mature SC.

Periodical striated patterns were described in animals, fungi and plants (see reviews by Westergaard and Wettstein, 1972; Zickler and Kleckner, 1999). When SCP3 is expressed in cultured somatic cells, cross-striated filaments with a periodicity of about $20 \mathrm{~nm}$ are formed (Yuan et al., 1998). This period approximately coincides with the spacing of the bands of the LEs found in some animals and in an Ascomycete (Zickler and Kleckner, 1999). The periodicity of the numerous short loops contained in the thickness of the ribbon-like structure of the LE is also about $20 \mathrm{~nm}$, although there is some dispersion of data. The DNA isolated and sequenced from purified SC was found to be different from random genomic sequences (Pearlman et al., 1992). It presents excess of microsatellites, mostly GT/CA repeats, and fragments of LINE and SINE sequences, which are interpreted as specialized attachment sequences (Pearlman et al., 1992; Moens, 1994; Moens et al., 1998). The loops of DNA lying inside the LE probably correspond to these attachment sequences associated with the proteins of the LE.

The irregular filament located at the central face of the LE of SC is stained with EDTA procedure and labeled by in situ hybridization carried out without the denaturation of the DNA of the sample. These features demonstrate that RNA is associated with the DNA-containing axial filament situated at the medial side of the LE in mature SC. The presence of the RNA in the fine axial chromatin filaments is detected short before these filaments are incorporated in the forming LE, and disappears progressively during the course of pachytene stage. It is difficult to ascertain the whether the filament located at the external side of the LE also contains RNA, due to its numerous loops and the multiple RNP structures, as perichromatin fibrils and perichromatin granules (Monneron and Bernhard, 1969), associated with it. The appearance of RNA bound to the chromosome axes during final pairing may represent the newly synthesized RNAs previously found using biochemical procedures and referred to as PsnRNA and zygRNA. A small nuclear RNA 125 nucleotides in length (PsnRNA) transcribed by RNA polymerase III was found to be synthesized during meiotic prophase in Lilium (Hotta and Stern, 1981). The transcription of a DNA replicated during chromosome pairing has been reported in lily and mouse meiocytes (Hotta et al., 1985). These transcripts (zygRNA) contain poly(A) and are found only in meiocytes (Hotta et al., 1985). Furthermore, autoradiographic experiments at the light microscope level have demonstrated nucleolar and extranucleolar transcription during meiotic prophase in various animals and plants (Taylor, 1959; Muckenthaler, 1964; Monesi, 1965; Kierszenbaum and Tres, 1974; Tres, 1975; Hartung and Stahl, 1976; Söderström, 1976; Geremia et al., 1977). The inhibition of transcription during meiotic prophase results in strong interference with meiotic process (Sakaguchi et al., 1983). When the inhibitor was applied in leptotene or zygotene, meiotic development arrests and synapsis does not take place (Sakaguchi et al., 1983). The authors suggest that late zygonema probably represents a physiological distinctive stage in meiotic development with respect to RNA metabolism which is related to the completion of synapsis. Taking into account all these results, the asso- 
ciation of RNA with the axial filaments before the formation of the SC and its presence in the chromatin axes of the LE during early pachytene is probably related to a still uncharacterized process essential for the achievement of final pairing. The intensity of the positive contrast of the forming leptotene and zygotene chromosomal axes with EDTA procedure for RNP is much higher than that of the lateral element of the forming SC. During pachytene stage the specific contrast due to RNA fades and finally disappears from the lateral elements of the SC. These observations lead us to speculate that the RNA is probably related to the assembly of the component of the axial element and its transformation to lateral element of the SC. Further work aimed at characterizing morphologically and cytochemical the structures associated with homology search and with the formation of the SCs is in progress. In order to interpret the presence of RNA in the SC it is important to study the localization of the transcriptional activity in several stages of meiotic prophase.

\section{ACKNOWLEDGMENTS}

This work was supported by grant I/74 348 of the Volkswagen-Stiftung and by UNAM, PAPIIT IN227398 grant. The authors are indebted with Ricardo Benavente for helpful and critical reading of the manuscript. We also wish to thank Mario Soriano for animal care and Porfirio Munguía Reyes for drawing the diagram.

\section{REFERENCES}

Baker S.M., Bronner C.E., Zhang L., Plug A.W., Robatzek M., Warren G., Elliott E.A., Yu J., Ashley T., Arnheim N., and Liskay M.: Male mice defective in the DNA mismatch repair gene PNS2 exhibit abnormal chromosome synapsis in meiosis. Cell 82, 309-319, 1995.

Baker T.G., and Franchi L.L.: The fine structure of oogonia and oocytes in human ovaries. J. Cell Sci. 2, 213-224, 1967.

Bernhard W.: A new staining procedure for electron microscopical cytology. J. Ultrastruct. Res. 27, 250-265, 1969.

Biggiogera M., Fakan S., Kaufmann S.H., Black A., Shaper J.H., and Bush H.: Simultaneous immunoelectron microscopic visualization of protein $\mathrm{B} 23$ and $\mathrm{C} 23$ distribution in the HeLa cell nucleolus. J. Histochem Cytochem. 9, 1371-1374, 1989.
Bishop D.K., Park D., Xu L., and Kleckner N.: DMC1: a meiosis-specific yeast homolog of $E$ coli recA required for recombination, synaptonemal complex formation, and cell cycle progression. Cell 69, 439-456, 1992.

Borde V., Goldman S.H., and Lichten M.: Direct coupling between meiotic replication and recombination initiation. Science 290, 806-809, 2000.

Carpenter A.T.C.: Electron microscopy of Drosophila melanogaster females. II. The recombination nodule: a recombination associated structure at pachytene? Proc. Nat. Acad. Sci. USA 72, 3186-3189, 1975.

Carpenter A.T.C.: Synaptonemal complex and recombination nodules in wild type of Drosophila melanogaster females. Genetics 92, 511-541, 1979a.

Carpenter A.T.C.: Recombination nodules and synaptonemal complex in recombination defective females of Drosophila melanogaster. Chromosoma 75, 259-292, 1979 b.

Clermont Y.: Cycle of the seminiferous epithelium of the guinea pig. Fert. Steril. 6, 563-573, 1960.

Cogliati R., and Gautier A.: Mise en évidence de l'ADN et des polysaccharides á l'aide d'un nouveau réactif de type Schiff. C. R. Acad. Sci. D 276, 3041-3044, 1973.

Coleman J, and Moses M.: DNA and the fine structure of synaptic structures in the domestic rooster. J. Cell Biol. 23, 63-78, 1964.

Esponda P., and Stockert J.C.: Localization of RNA in the synaptonemal complex. J. Ultrastruct. Res. 35, 411-417, 1971.

Fawcett D.W.: The fine structure of chromosomes in the meiotic prophase of vertebrate spermatocytes. J. Biophys. Biochem. Cytol. 2, 403-406, 1956.

Geremia R., Boitani C., Conti M. and Monesi V.: RNA synthesis in spermatocytes and spermatids and preservation of meiotic RNA during spermiogenesis in the mouse. Cell Differentiation 5, 343-355, 1977.

Hartung H., and Stahl A.: Incorporation of tritiated uridine during pachytene and diplotene stages in the oocytes of the japanese quail (Coturnix coturnix japonica). Experientia 32, 96-97, 1976.

Hawley R.S., and Arbel T.: Yeast genetics and the fall of the classical view of meiosis. Cell 72, 301-303, 1993.

Hotta Y., and Stern H.: Small nuclear RNA molecules that regulate nuclease accessibility in specific chromatin region of meiotic cells. Cell 27, 309-319, 1981.

Hotta Y., Tabata S., Stubbs L., and Stern H.: Meiosis-specific transcripts of a DNA component replicated during chromosome pairing: homology across the phylogenetic spectrum. Cell 40, 785-793, 1985.

Kierszenbaum A.L., and Tres L.L.: Transcription sites in spread meiotic prophase chromosomes from mouse spermatocytes. J. Cell Biol. 63, 923-925, 1974.

Kleckner N.: Meiosis: How could it work? Proc. Nat. Acad. Sc. USA 93, 8167-8174, 1996. 
Kleckner N., Padmore R., and Bishop D.K.: Meiotic chromosome metabolism: one view. Cold Spring Harbor Sym. Quant. Biol. 56, 729-743, 1991.

Leblond C.P., and Clermont Y.: Definition of the stages of the cycle of the seminiferous epithelium in the rat. Ann. New York Acad. Sci. 55, 548-569, 1952.

Maguire M.P.: Is the synaptonemal complex a disjunction machine? J. Heredity 86, 330-340, 1995.

McKim K., Green-Marroquin B.L., Sekelsky J.J., Chin G., Steinberg C., Khodosh R., and Hawley R.S.: Meiotic synapsis in the absence of recombination. Science 279, 876-878, 1998.

Moens P.B.: Molecular perspectives of chromosome pairing at meiosis. BioEssays 16, 101-106, 1994.

Moens P.B., Pearlman R.E., Heng H.H.Q., and Traut W.: Chromosome cores and chromatin at meiotic prophase. Curr. Topics Develop. Biol. 37, 241-262, 1998.

Monneron A., and Bernhard W.: Fine structural organization of the interphase nucleus of some mammalian cells. J. Ultrastruct. Res. 27, 266 - 288, 1969.

Monesi V.: Synthetic activities during spermatogenesis in the mouse. RNA and protein. Exp. Cell Res. 39, 197-224, 1965.

Moses M.J.: Chromosomal structures in crayfish spermatocytes. J. Biophys. Biochem. Cytol. 2, 215-218, 1956.

Moses M.J.: Synaptinemal complex. Ann. Rev. Genet. 2, 363 $412,1968$.

Muckenthaler F.A.: Autoradiographic study of nucleic acids synthesis during spermatogenesis in the grasshoper, Melanoplus differentialis. Exp. Cell Res. 35, 531-547, 1964.

Nebel B.R., and Coulon E.M.: The fine structure of chromosomes in pigeon spermatocytes. Cromosoma 13, 272-291, 1962.

Padmore R., Cao L., Klekner N.: Temporal comparison of recombination and synaptonemal complex formation during meiosis in S. cerevisae. Cell 66, 1239-1256, 1991.

Pearlman R.E., Tsao N., and Moens P.B.: Synaptonemal complexes from DNase-treated rat pachytene chromosomes contain $(\mathrm{GT})_{\mathrm{n}}$ and LINE sequences, but no MARs/SARs. Genetics $130,865-872,1992$.

Roeder G.S.: Sex and the single cell: Meiosis in yeast Proc. Nat. Acad. Sci. USA 92, 10450-10456, 1995.

Sakaguchi K., Miyako H., Takegami M.H., and Ito M.: Inhibition of RNA synthesis in meiotic cells and its effect on meiotic development. Cell Struct. Funct. 8, 127-135, 1983.

Scherthan H., Loidl J., Schuster T., and Schweitzer D.: Meiotic chromosome condensation and pairing in Saccharomyces cerevisiae studied by chromosome painting. Chromosoma 101, 590-595, 1992.

Söderström K.O.: Characterization of RNA synthesis in midpachytene spermatocytes of the rat. Exp. Cell Res. 102, 327245, 1976.

Sotelo J.R.: Ultrastructure of the chromosomes at meiosis. In Handbook of Molecular Cytology. (Ed. Lima de Faria A.) North-Holland Pub. Co., Amsterdam, pp. 412-434, 1969.
Sotelo J.R., and Trujillo-Cenoz O.: Microscopic structure of meiotic chromosomes during prophase. Exp. Cell Res. 14, 18, 1958.

Sym M., Engebrecht J., and Roeder G.S.: ZIP1 is a synaptonemal complex protein required for meiotic chromosome synapsis. Cell 72, 365-378, 1993

Taylor J.H.: Autoradiographic studies of nucleic acids and proteins during meiosis in Lilium longiflorum. Am. J. Botany 46, 477-484, 1959.

Tres L.L.: Nucleolar RNA synthesis of meiotic prophase spermatocytes in the human testis. Chromosoma 53, 141-151, 1975.

Vázquez-Nin G.H., Biggiogera M., and Echeverría O.M.: Activation of osmium ammine by $\mathrm{SO}_{2}$-generating chemicals for electron microscopy Feulgen-type staining of DNA. Eur. J. Histochemistry 39, 101-106, 1995.

Vázquez-Nin G.H., and Echeverría O.M.: Ultrastructural study on meiotic prophase nucleus of rat oocytes. Acta Anat. 96, 218-231, 1976.

Vázquez-Nin G.H., Echeverría O.M., Fakan S., Leser G., and Martin T.E.: Immunoelectron microscope localization of snRNPs in the polytene nucleus of salivary glands of Chironomus thummi. Chromosoma 99, 44-51, 1990.

Vázquez-Nin G.H., Flores E., Echeverría O.M., Merkert H., Benavente R., and Wettstein R.: Immunocytochemical localization of DNA in synaptonemal complexes of rat and mouse spermatocytes and chick oocytes. Chromosoma 102, 457-463, 1993.

Watson M.L.: Spermatogenesis in the adult albino rat as revealed by tissue sections in the electron microscope. Univ. Rochester Atomic Energy Report UR-185, 1952.

Westergaard M., and Wettstein D.: The synaptonemal complex Ann. Rev. Genet. 6, 71-110, 1972.

Wettstein R., and Sotelo J.R.: Electron microscope serial reconstruction of the spermatocyte I nuclei at pachytene. J. Microscop. 6, 557-576, 1967.

Wolstenholme D.R., and Meyer G.F.: Some facts concerning the nature and formation of axial core structures in spermatids of Gryllus domesticus. Chromosoma 18, 272-286, 1966.

Yuan L., Pelttari J., Brundell E., Bjorkroth B., Zhao J., Liu J.G., Brismar H., Daneholt B., and Hoog C.: The synaptonemal complex protein SCP3 can form multistranded cross-striated fibers in vivo. J. Cell Biol. 142, 331-339, 1998.

Zickler D., and Kleckner N.: Meiotic chromosomes: integrating structure and function. Annu. Rev. Genet. 33, 603-754, 1999. 